University of Wollongong

Research Online

Australian Institute for Innovative Materials -

Papers

Australian Institute for Innovative Materials

$1-1-2016$

Characteristics and cadmium extraction performance of PVC/Aliquat 336 electrospun fibres in comparison with polymer inclusion membranes

Nurul Syazana Binti Abdul Halim

University of Wollongong, University malaysia, nsbah996@uowmail.edu.au

Philip G. Whitten

University of Wollongong, whitten@uow.edu.au

Long D. Nghiem

University of Wollongong, longn@uow.edu.au

Follow this and additional works at: https://ro.uow.edu.au/aiimpapers

Part of the Engineering Commons, and the Physical Sciences and Mathematics Commons

Research Online is the open access institutional repository for the University of Wollongong. For further information contact the UOW Library: research-pubs@uow.edu.au 


\title{
Characteristics and cadmium extraction performance of PVC/Aliquat 336 electrospun fibres in comparison with polymer inclusion membranes
}

\author{
Abstract \\ Electrospun fibres and polymer inclusion membranes (PIMs) were prepared from polyvinyl chloride (PVC) \\ and Aliquat 336. Morphological and thermomechanical properties of the electrospun mats differed \\ notably from those of PIMs. The plasticizing effect of Aliquat 336 on electrospun PVC/Aliquat 336 fibres \\ was confirmed by the shifting of the glass transition temperature (Tg). By contrast, Aliquat 336 did not act \\ as a plasticizer in PIMs as Tg was independent of Aliquat 336 concentration. Cadmium extraction to \\ electrospun fibres could occur at a lower Aliquat 336 content (i.e. 6 wt.\%) compared with PIMs. At 40 \\ wt.\% Aliquat 336 content, both PIMs and electrospun fibrous mats exhibited similar extraction rate.

\section{Keywords} \\ fibres, membranes, electrospun, 336, inclusion, aliquat, cadmium, pvc, polymer, performance, comparison, \\ extraction, characteristics \\ Disciplines \\ Engineering | Physical Sciences and Mathematics \\ Publication Details \\ Abdul Halim, N., Whitten, P. G. \& Nghiem, L. D. (2016). Characteristics and cadmium extraction \\ performance of PVC/Aliquat 336 electrospun fibres in comparison with polymer inclusion membranes. \\ Separation Science and Technology (Philadelphia), 51 (9), 1515-1522.
}




\title{
FULL TITLE: Characteristics and cadmium extraction performance of PVC/Aliquat 336 electrospun fibres in comparison with polymer inclusion membranes
}

\author{
Submitted to \\ Separation Science and Technology
}

March 2015

Nurul Syazana Abdul-Halim ${ }^{1,2}$, Philip G. Whitten ${ }^{3}$ and Long D. Nghiem ${ }^{2, *}$

${ }^{1}$ Faculty of Earth Science

Universiti Malaysia Kelantan Kampus Jeli, Karung Berkunci No.100, 17600 Jeli, Kelantan, Malaysia.

${ }^{2}$ Strategic Water Infrastructure Laboratory School of Civil, Mining and Environmental Engineering University of Wollongong, NSW 2522, Australia

${ }^{3}$ Intelligent Polymer Research Institute, ARC Centre of Excellence for Electromaterials Science, AIIM Facility, University of Wollongong, NSW 2522 Australia 


\section{Abstract}

29 Electrospun fibres and polymer inclusion membranes (PIMs) were prepared from polyvinyl 30 chloride (PVC) and Aliquat 336. Morphological and thermal analysis showed that 31 electrospun fibres have different properties compared to those of PIMs with similar PVC and 32 Aliquat 336 composition. Electrospun PVC/Aliquat 336 fibrous mats have a porous web like 33 structure while PVC/Aliquat 336 PIMs are a pore free solid. The thermal analysis results 34 indicate that Aliquat 336 has a different plasticising effect on the electrospun fibres and 35 PIMs. The plasticising effect of Aliquat 336 on the electrospun PVC/Aliquat 336 fibres was 36 confirmed by a single glass transition temperature $\left(\mathrm{T}_{\mathrm{g}}\right)$ shifted towards lower temperature. 37 While PVC/Aliquat 336 PIMs consist of two or more phases as both distinct $\mathrm{T}_{\mathrm{g}}$ and melting 38 temperatures $\left(\mathrm{T}_{\mathrm{m}}\right)$ are present in DMA analysis. Both PVC/Aliquat 336 fibres and PIMs 39 could be used for cadmium extraction. However, cadmium extraction to electrospun fibres was higher than that observed for PIMs and could occur at a low Aliquat 336 content (i.e. 6

41 wt.\%). By contrast, there appears to be a percolation threshold of Aliquat 336 in PIMs of 30 wt.\% when cadmium extraction started to occur.

43 Keywords: Electrospun fibres; polymer inclusion membranes (PIMs); metal extraction; 44 thermal analysis; polyvinyl chloride (PVC); Aliquat 336. 


\section{Introduction}

The rapid development of modern industries such as electroplating, electronic production, solar photovoltaic and batteries has significantly heightened the interest in heavy metal extraction. In fact, heavy metals are essential for these industries. For example, cadmium is extensively employed for the production of batteries, pigmentation, electronic components, and nuclear power amongst many other industries [1,2]. Cadmium is primarily produced from mineral ores and often a by-product during the refining zinc and lead. Thus, the extraction of heavy metals is of significant interest to the mineral processing industry. The disposal or accidental release of heavy metals to the environment is also of significant concern. Unlike organic contaminants, heavy metals are not biodegradable and tend to accumulate in living organisms. Many heavy metals are known to be toxic or carcinogenic. Toxic heavy metals of particular concern in treatment of industrial wastewaters include cadmium, zinc, copper, nickel, mercury, lead and chromium. Thus, the extraction of heavy metals from contaminated water is also of significant interest for environmental protection.

The extraction of most heavy metals including cadmium has been traditionally carried out by solvent-solvent extraction. However, this technique is energy intensive and uses a significant volume of organic diluents which are volatile, flammable and harmful to human health and the environment [3]. Polymer inclusion membranes (PIMs) present an alternative approach to recover heavy metals from an aqueous solution that potentially requires a smaller physical footprint and is significantly more environmentally friendly than solvent-solvent extraction. PIMs are a type of liquid membrane that relative to conventional liquid membranes exhibit longer process lifetimes, superior mechanical properties and higher chemical stability $[4,5]$. In addition, PIMs allow for simultaneous extraction and stripping of the target ion and thus accelerate the separation process compared to conventional solvent extraction which can only be used in batch mode [4]. Generally, PIMs consist of polyvinyl chloride (PVC) or cellulose triacetate (CTA) as a base polymer, an extractant, and a plasticizer. Numerous studies have shown effective metal ions extraction using PIMs [4, 6-12]. In addition, the use of PIMs for sample preparation [13] and low cost sensory devices [14] has recently been demonstrated.

Electrospinning is an innovative technique for the production of polymer fibres with diameter of less than a few micrometres, resulting in a large surface area-to-volume ratio and high porosity. These polymer fibres can potentially be used in numerous applications including tissue engineering, wound dressing, and drug delivery [15]. A few research groups [16, 17] have successfully developed polymer fibres using the electrospinning method for heavy metal 
extraction from aqueous solutions. Similar to the preparation of PIMs, a solution is first prepared by incorporating polymer and extractant using solvent. Then the solution is electrospun using electrospinning equipment at certain parameters to produce the mats consisting of electrospun fibres. Wong et al. [17] reported that the extraction of cadmium has improved using electrospun PVC/Aliquat 336 compared to film cast PVC/Aliquat 336 PIMs. However, Wong et al. [17] did not to characterise the electrospun fibres and thus little is known about the influence of their thermomechanical properties on metal extraction.

In this study, electrospun fibrous mats and PIMs were fabricated from PVC at various Aliquat 336 concentrations. The effects of Aliquat 336 content on the morphological structure as well as thermal properties of electrospun fibrous mats were investigated using scanning electron microscopy (SEM) and dynamic mechanical analysis (DMA). The physical properties of electrospun PVC/Aliquat 336 mats were then compared to those of PIMs to explain for the performance of these materials with respect to heavy metal extraction. The results provide insights for further development of electrospun PVC/Aliquat 336 fibres for heavy metal extraction.

\section{Materials and methods}

\subsection{Reagents}

High molecular weight PVC from Sigma Aldrich (Australia) was used as the base polymer. The weight-average molecular weight of this PVC is $80,000 \mathrm{~g} / \mathrm{mol}$. Aliquat 336 (tricaprylmethylammonium chloride) from Sigma Aldrich (Australia) was used as the extractant. Aliquat 336 is a mixture of tri-alkyl methyl ammonium chloride salts produced from the methylation of Alamine 336, with the substituent alkyl chain length containing between 6 and 12 carbon atoms. HPLC grade tetrahydrofuran (THF) and dimethylflormamide (DMF) from BDH (Australia) were used without any further purification. Cd(II) was selected as a model heavy metal. $\mathrm{Cd}$ (II) solutions used in the extraction experiments were prepared from analytical grade $\mathrm{Cd}\left(\mathrm{NO}_{3}\right)_{2}$. Milli-Q grade water (Millipore, Australia) was used for the preparation of all aqueous solutions.

\subsection{Preparation of PVC/Aliquat 336 fibres}

Electrospun mats at different Aliquat 336 concentrations were prepared from PVC and Aliquat 336. The electrospinning apparatus consisted of a high voltage supply (Gamma Model ES30P-5W/DAM, Gamma High Voltage Research Inc.), a syringe pump (KDS 100, 


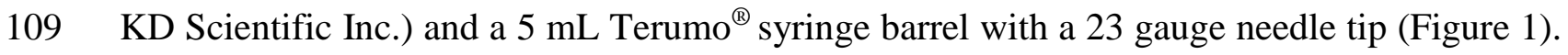

110 About $3 \mathrm{~mL}$ of the prepared solution was used to form an individual mat.

111 Several electrospinning conditions (in terms of flow rate, voltage, and distance to the

112 collector) were evaluated to determine parameters that would result in consistent fibre

113 formation. The optimised electrospinning conditions were then used in this study. Briefly, the

114 polymer solution was electrospun at $0.06 \mathrm{~mL} / \mathrm{h}$ with an applied voltage of $17 \mathrm{kV}$, and

115 distance between the syringe tip and the aluminium collector of $170 \mathrm{~mm}$.

116 PVC/Aliquat 336 solutions were first prepared by dissolving Aliquat $336(0-40 \mathrm{wt} . \%)$ and 117 PVC (100-60 wt.\%) in THF/DMF solvent (1:1 by volume). During the electrospinning 118 process, due to the applied voltage, the charged polymer could overcome the surface tension 119 of the solution. Thus, a charged polymer jet was ejected from the needle tip and deposited on 120 the grounded aluminium collector as polymer fibres. The fibrous mats were collected after 5 121 hours of electrospinning.

\section{2.3. Preparation of PVC/Aliquat 336 PIMs}

124 PIMs at different Aliquat 336 concentrations were prepared by dissolving Aliquat 336 and PVC in THF without DMF as described previously [18]. Each mixture contained a combined Aliquat 336 and PVC weight of $600 \mathrm{mg}$. The volume of THF used was between 5 to $10 \mathrm{~mL}$ depending on the weight fraction of PVC. The mixtures were stirred vigorously for 1 hour resulting in a clear solution. The solution was then transferred into a Petri dish with a diameter of $70 \mathrm{~mm}$ and covered with filter paper. The THF solvent was allowed to evaporate over about 48 hours to form PIMs. The membranes were peeled from the Petri dish and stored in the dry condition for further experiments. PVC films were prepared using the same protocol but without the addition of Aliquat 336.

\subsection{Scanning electron microscopy}

134 Morphology of the electrospun fibrous mats and PIMs was characterized using a scanning electron microscope (SEM) (JEOL JCM 6000). Square sections of samples approximately 10 $\mathrm{mm}$ by $10 \mathrm{~mm}$ were mounted on aluminium stubs. The samples were then gold coated using

137 a DYNAVAC Mini Coater prior to SEM analysis. For electrospun fibrous mats, fibre 138 diameters were determined by Image $\mathrm{J}$ software. A minimum of six fibre diameter measurements were conducted for each condition. 


\subsection{Dynamic mechanical analysis}

141 A DMA Q 800 (TA Instrument, USA) was used to characterise the thermal transitions of 142 electrospun fibre mats and PVC/Aliquat 336 PIMs. A tensile film-clamp was used with a 143 heating rate of $4{ }^{\circ} \mathrm{C} / \mathrm{min}$ over the temperature range of -70 to $110{ }^{\circ} \mathrm{C}$ at a frequency of $1 \mathrm{~Hz}$. 144 For DMA, the approximate distance between clamps was $15 \mathrm{~mm}$, and the oscillation 145 displacement was $10 \mu \mathrm{m}$. The temperatures associated with transitions were identified by the 146 maximum in the corresponding tan delta curve. Where defined, the thermal transitions are

147 labelled in order from highest to lowest temperature.

\subsection{Cadmium extraction protocol}

149 Extraction experiments were conducted in bath mode using a protocol described in details 150 elsewhere [18]. PIMs or electrospun fibrous mats with membrane mass of $0.57 \pm 0.01 \mathrm{~g}$ and $1510.03 \pm 0.01 \mathrm{~g}$ respectively were placed in beakers containing $50 \mathrm{~mL}$ of extraction solution. 152 For the electrospun mats, the extraction solution contained $3 \mathrm{mg} / \mathrm{L}$ of $\mathrm{Cd}(\mathrm{II})$ in $1 \mathrm{M}$ 153 hydrochloric acid $(\mathrm{HCl})$ whereas for the PIMs, the extraction solution contained $50 \mathrm{mg} / \mathrm{L}$ of $154 \mathrm{Cd}(\mathrm{II})$ in $1 \mathrm{M}$ hydrochloric acid $(\mathrm{HCl})$. These different extraction solution concentrations were used to obtain the same ratio of membrane mass to cadmium concentration. The extraction solution was stirred continuously with a $1 \mathrm{~mL}$ of aliquot was taken at specific time intervals for metal ion analysis using Atomic Adsorption Spectrometry analysis (Varian

158 SpectrAA 300 AAS, Australia). Calibration using standard Cd(II) solutions was conducted 159 prior to each batch of analysis. The linear regression coefficients for all calibration curves 160 were greater than 0.98 .

\section{3. Results and discussion}

\subsection{Membrane preparation and thickness}

163 The electrospun PVC/Aliquat 336 fibrous mats obtained from this study were opaque 164 regardless of the Aliquat 336 content which was from 0 to 40 wt.\%. By contrast, the 165 PVC/Aliquat 336 PIMs were transparent, homogenous, and flexible (Figure 1a). The opacity 166 of the electrospun fibrous mat was expected and is due to light scattering from free fibre 167 surfaces. It is noteworthy that the electrospun PVC/Aliquat 336 mat was thicker at the centre 168 of the collector relative to the edge while the thickness of PVC/Aliquat 336 PIMs was relatively uniform over the entire area. 


\subsection{Membrane surface morphology}

172 A fibrous web like structure could be seen with all PVC/Aliquat 336 electrospun fibre mats 173 obtained from this study (Figure 2b-f). Electrospun fibres obtained from only PVC were 174 small, densely packed, and uniform (Figure 2b). As the Aliquat 336 content increased from 6 to $40 \mathrm{wt} . \%$, the electrospun fibre diameter within the mat became less uniform (Figure 2b-f). In addition, the fibres diameter increased from about $1.5 \pm 0.2 \mu \mathrm{m}$ (without any Aliquat 336) to $3.5 \pm 0.3 \mu \mathrm{m}$ at $40 \mathrm{wt} \%$ Aliquat 336. As the Aliquat 336 content increased beyond 12 wt.\%, the formation of beads occured. The number and size of these beads appear to increase monotonically with the Aliquat 336 content. Beads are common in electrospinning and are related to the instability of the polymer solution jet [19]. The formation of beads can be reduced by changing the polymer concentration, surface tension, flow rate, distance between tip and collector, and, voltage [20]. In this study, the same set of electrospinning parameters as explained in section 2.2 was used for all membrane compositions.

Unlike the electrospun fibrous mats, all PVC/Aliquat 336 PIMs were transparent and have a non-porous surface (Figure 3). At 10 to 20 wt.\% Aliquat 336, the PIM surface was featureless (Figure 3b-c). However at $30 \mathrm{wt}$ \% Aliquat 336, some wrinkles could be observed (Figure 3d). The surface wrinkles are more regular at 40 wt.\% Aliquat 336 (Figure 3e). The wrinkles are probably formed due to a THF concentration gradient during film formation. Typically, during the early stage of membrane formation the THF at the free surface will evaporate readily forming a membrane skin. Below this skin there is a relatively high THF concentration that over time diffuses through the skin with the formation of the membrane. Coinciding with the evaporation of THF is a reduction in volume, which places a compressive force on the membrane surface resulting in wrinkles. Also coinciding with a reduction in THF is the conversion of a solution to a mixture containing a PVC rich phase and a Aliquat 336 rich phase [21]. The Aliquat 336 concentration dependence of wrinkle formation could be attributed to either a lower membrane elastic modulus or the Aliquat 336 rich phase. The former provides a lower buckling stress while the latter retains a large volume fraction of THF relative to the PVC rich phase in the preliminary stage of film formation. 


\subsection{Membrane thermal analysis}

203 DMA is a complimentary method for assessing the thermo-mechanical properties of 204 polymeric materials. Figures 4 and 5 show the storage modulus and $\tan \delta$ of electrospun 205 PVC/Aliquat 336 and PVC/Aliquat 336 PIMs at different Aliquat 336 content.

206 DMA results revealed that electrospun PVC/Aliquat 336 and PVC/Aliquat PIMs exhibited $\alpha$ transitions which were determined to be the $T_{g}$ values. The $T_{g}$ value of electrospun PVC without Aliquat 336 was $98{ }^{\circ} \mathrm{C}$. This is consistent with the value reported for pure PVC in the literature [22]. However, the $\mathrm{T}_{\mathrm{g}}$ of PVC PIMs without Aliquat 336 was $67{ }^{\circ} \mathrm{C}$ which is lower than that of pure PVC $[18,23,24]$. The depression of $\mathrm{T}_{\mathrm{g}}$ observed is possibly due to the small amount of residual THF in the PVC/Aliquat 336 PIMs [21]. Hence, the THF/DMF solvent used for electrospun PVC/Aliquat 336 is likely to have completely evaporated during the electrospinning process.

214 The $\alpha$ transition of electrospun PVC/Aliquat 336 has shifted to a lower temperature as 215 Aliquat 336 content increased from 0 to $40 \mathrm{wt}$ \% (Figure $4 \mathrm{a}$ and 5a). This result indicates that 216 Aliquat 336 may contribute to the PVC segmental mobility of the electrospun mats or that the retained stress from electrospinning is relative to the Aliquat 336 concentration. In contrast, constant values of $\alpha$ transition were observed for PVC/Aliquat 336 PIMs containing 0 to 40 wt.\% Aliquat 336 (Figure 4b and 5b).

The $\beta$ transition was observed at $-18{ }^{\circ} \mathrm{C}$ for PVC/Aliquat 336 PIM containing 40 wt.\% Aliquat 336 (Figure $4 \mathrm{~b}$ and $5 \mathrm{~b}$ ). The $\beta$ transition is assigned to the melting temperature $\left(\mathrm{T}_{\mathrm{m}}\right)$ of Aliquat 336 as it is consistent with the report value of $-20^{\circ} \mathrm{C}$ [25].

The addition of Aliquat 336 in PVC formed by electrospinning induced a decrease of $\mathrm{T}_{\mathrm{g}}$ values (Figure 4a). In other words, Aliquat 336 has plasticized the electrospun fibres or THF that may be retained in the Aliquat which subsequently plasticizes the PVC phase of the fibres. By contrast, in the solvent cast PVC based PIM system, the $\mathrm{T}_{\mathrm{g}}$ value was independent of Aliquat 336 concentration over the range of 0 to $40 \mathrm{wt} . \%$ indicating that PVC segmental mobility is not a funtion Aliquat 336 concentration [18].

A single transition observed with all PVC/Aliquat 336 electrospun fibres (Figure 4a and 5a)

230 indicates that they are homogenous or that the PVC and Aliquat 336 are not phase separated.

231 Alternatively, the elastic modulus of the electrospun mats is an order of magnitude lower than 232 that of the PIMs. This substantially low elastic modulus is due to the high porosity and is a 
233 function of fibre or fibre junction stiffness [26]. Hence, it is possible that the DMA used here

234 is not sensitive to the beta transition in electrospun fibrous mats if it is present. On the other 235 hand, PVC/Aliquat 336 PIMs containing 20 to 40 wt.\% Aliquat 336 are phase separated with 236 two discrete phases rich in PVC and Aliquat 336 (Figure 4b and 5b). Even though the 237 PVC/Aliquat 336 PIMs at 20 and 30 wt.\% Aliquat 336 did not exhibit any $\beta$ transition 238 (Figure 4b) but a decreased in storage modulus at about $-22{ }^{\circ} \mathrm{C}$ indicated that they also 239 contain Aliquat 336 rich phase. However, there is no indication of an Aliquat 336 rich phase 240 in PIMs containing 10 wt.\% Aliquat 336 by DMA. Overall, the storage modulus of 241 PVC/Aliquat 336 electrospuns mats were much lower than PVC/Aliquat 336 PIMs but the values increased as the Aliquat 336 content increased (Figure 4a).

243 It is also noteworthy that the transparency of all the electrospun fibrous mats had changed 244 during the DMA experiment. Specifically, the regions of the electrospun fibrous mat that were within and adjacent to the DMA clamps changed from opaque to transparent during a DMA heating sequence. Hence, the combination of temperature and pressure was sufficient to achieve viscous flow of the polymer with the electruspun fibres fusing to form a solid. The central section of the electrospun mat remained opaque, even after heating to $110^{\circ} \mathrm{C}$.

\section{[FIGURE 4]}

\section{[FIGURE 5]}

\subsection{Cadmium extraction performance}

252

253

254

255

256

257

258

259

260

The extraction kinetics of $\mathrm{Cd}(\mathrm{II})$ to PVC/Aliquat 336 electrospun fibrous mats and PIMs are shown in Figure 6. As expected, in the absence of Aliquat 336 (which was used as the extraction), no extraction of $\mathrm{Cd}(\mathrm{II})$ to electrospun mats and PIMs could be observed. As can be seen from Figure 6a, the extraction rate of PVC/Aliquat 336 electrospun fibrous mats increased when Aliquat 336 content increased. There was a significant increase of $\mathrm{Cd}(\mathrm{II})$ removal for electrospun PVC/Aliquat 336 mats containing 12 to $40 \mathrm{wt} \%$ Aliquat 336 (Figure 6a). Maximum extraction was reached at 25 and 40 wt.\% Aliquat 336 with about $98 \%$ removal of $\mathrm{Cd}(\mathrm{II})$.

By comparison, the extraction of Cd(II) using PIMs was not significant at low Aliquat 336 content (Figure 6b). A significant extraction was observed in PIMs containing 30 wt.\% Aliquat 336 where a major change in $\mathrm{Cd}(\mathrm{II})$ removal was witnessed. However the extraction was only $72 \%$ completed. This result is in good agreement with the data reported by Xu et al. 
264 [11] that the extraction is not viable for PIMs containing less than $30 \mathrm{wt} . \%$ of Aliquat 336.

265 Maximum extraction was reached at 40 wt.\% Aliquat 336 with 95\% $\mathrm{Cd}(\mathrm{II})$ removal which coincides with the appearance of a defined $\beta$ transition (Aliquat 336 rich phase).

267 The extraction capacity was also plotted against Aliquat 336 concentrations for both 268 electrospun fibres and PIMs as shown in Figure 7. The extraction capacity can be calculated 269 by using the equation as described below:

270 Extraction capacity $(\mathrm{mg} / \mathrm{g})=\frac{\left[V_{i}-V_{f}\right] \times V_{s}}{M}$

271 where $V_{i}$ and $V_{f}$ are the initial and final concentration of $\mathrm{Cd}(\mathrm{mg} / \mathrm{L})$ respectively. $V_{s}$ is the starting of the feed volume (L) and $M$ is the mass of the membrane (g).

273 Base on the result, the extraction capacity of both membranes has reached more than $4 \mathrm{mg} / \mathrm{g}$ at 40 wt. \% of Aliquat 336 content. However, in all cases, electrospun fibrous mats has higher extraction capacity even at low Aliquat 336 content compared to PIMs. The increasing of

$276 \mathrm{Cd}(\mathrm{II})$ removal for electrospun fibrous mats is possibly due to the increasing of surface area 277 containing Aliquat 336. As noted in section 3.2, electrospun mats revealed a homogenous 278 web like structure. Therefore, electrospun fibrous mats have larger surface area containing 279 Aliquat 336 than PIMs and hence improved the Cd(II) removal.

280 On the other hand, PVC/Aliquat 336 PIMs has distinctive PVC and Aliquat 336 rich phases. 281 For heterogeneous membrane, the transport of metal ions requires continuous channels. 282 Unlike electrospun fibres, sufficient amount of extractant is essential to form continuous 283 channels across the PIMs for the extraction to occur. In this study the percolation threshold was observed to be at $30 \mathrm{wt} . \%$ Aliquat 336.

[FIGURE 6]

\section{[FIGURE 7]}

\section{$287 \quad 4 . \quad$ Conclusion}

288 In this study, the properties and Cd(II) extraction performance of PVC/Aliquat 336 fibrous mats and PIMs prepared by electrospinning and conventional casting, respectively, were evaluated and compared. The results showed that the role of Aliquat 336 in electrospun fibres differ from that in PIMs. The PVC/Aliquat 336 electrospun fibrous mats exhibited web like structures and were visually opaque. However, electrospun fibres were homogenous and have 
only a single phase that is $\alpha$ transition. The electrospun fibres were plasticized by Aliquat 336 since the $T_{g}$ value observed by DMA decreased with the increasing of Aliquat 336 content. On the other hand, the PVC/Aliquat 336 PIMs were visually transparent but were phase separated with two distinct phases that is $\alpha$ transition and $\beta$ transition observed by DMA. In addition, Aliquat 336 did not act as a plasticizing agent in the PIM system since the $\mathrm{T}_{\mathrm{g}}$ did not decreased as the Aliquat 336 content increased. The extraction kinetics of $\mathrm{Cd}(\mathrm{II})$ to both PVC/Aliquat 336 electrospun fibrous mats and PIMs increased when Aliquat 336 content increased. However, the extraction of $\mathrm{Cd}(\mathrm{II})$ to PVC/Aliquat 336 PIMs was dependent on the appearance of the defined $\beta$ transition. To facilitate cadmium extraction, the Aliquat 336 content in PIMs needs to exceed the percolation threshold of $30 \mathrm{wt} \%$. By contrast, cadmium extraction to electrospun fibrous mats could occur at a much lower Aliquat 336 content (i.e. 6 wt.\%).

\section{References}

[1] Bertin, G. and D. Averbeck, Cadmium: cellular effects, modifications of biomolecules, modulation of DNA repair and genotoxic consequences (a review). Biochimie, 2006. 88(11): p. 1549-1559.

[2] Wang, X.S., Z.Z. Li, and S.R. Tao, Removal of chromium (VI) from aqueous solution

[3] Sgarlata, C., R.A. Bartsch, E. Longo, G. Arena, D. Zhang, and Y. Yang, Heavy metal separation with polymer inclusion membranes. Journal of Membrane Science, 2008. 323(2): p. 444-451.

[4] Nghiem, L.D., P. Mornane, I.D. Potter, J.M. Perera, R.W. Cattrall, and S.D. Kolev, Extraction and transport of metal ions and small organic compounds using polymer inclusion membranes (PIMs). Journal of Membrane Science, 2006. 281(1-2): p. 7.

[5] Kagaya, S., Y. Ryokan, R.W. Cattrall, and S.D. Kolev, Stability studies of poly(vinyl chloride)-based polymer inclusion membranes containing Aliquat 336 as a carrier. Separation and Purification Technology, 2012. 101: p. 69-75.

[6] Gherasim, C.-V.I., G. Bourceanu, R.-I. Olariu, and C. Arsene, Removal of lead(II) from aqueous solutions by a polyvinyl-chloride inclusion membrane without added plasticizer. Journal of Membrane Science, 2011. 377(1-2): p. 167-174.

[7] Almeida, M.I.G.S., R.W. Cattrall, and S.D. Kolev, Recent trends in extraction and transport of metal ions using polymer inclusion membranes (PIMs). Journal of Membrane Science, 2012. 415-416(0): p. 9-23.

[8] Gherasim, C.V., G. Bourceanu, R.I. Olariu, and C. Arsene, A novel polymer inclusion membrane applied in chromium (VI) separation from aqueous solutions. Journal of Hazardous Materials, 2011. 197: p. 244-253. 
[9] Regel-Rosocka, M., Ł. Nowak, and M. Wiśniewski, Removal of zinc(II) and iron ions from chloride solutions with phosphonium ionic liquids. Separation and Purification

[10] Benosmane, N., S.M. Hamdi, M. Hamdi, and B. Boutemeur, Selective transport of metal ions across polymer inclusion membranes (PIMs) containing calix[4]resorcinarenes. Separation and Purification Technology, 2009. 65(2): p. 211.

[11] Pospiech, B., Synergistic Solvent Extraction and Transport of $\mathrm{Zn}$ (II) and $\mathrm{Cu}$ (II) across Polymer Inclusion Membranes with a Mixture of TOPO and Aliquat 336. Separation Science and Technology, 2014. 49(11): p. 1706-1712.

[12] Raut, D.R. and P.K. Mohapatra, A novel PVC based polymer inclusion membrane containing TODGA as the extractant for the pre-concentration of americium from acidic feed solutions. Separation Science and Technology, 2013. 48(16): p. 24992505.

[13] Ohshima, T., S. Kagaya, M. Gemmei-Ide, R.W. Cattrall, and S.D. Kolev, The use of a polymer inclusion membrane as a sorbent for online preconcentration in the flow injection determination of thiocyanate impurity in ammonium sulfate fertilizer. Talanta, 2014. 129: p. 560.

[14] Fontàs, C., R. Vera, A. Batalla, S. Kolev, and E. Anticó, A novel low-cost detection method for screening of arsenic in groundwater. Environmental Science and Pollution Research, 2014. 21(20): p. 11682-11688.

[15] Frenot, A. and I.S. Chronakis, Polymer nanofibers assembled by electrospinning. Current Opinion in Colloid \& Interface Science, 2003. 8(1): p. 64-75.

[16] Truong, Y.B., I.L. Kyratzis, and W. Shen, Fabrication and characterization of electrospun PVDF-aliquat 336 fibre membrane for removal of cadmium from hydrochloric acid solutions. Journal of Materials Science, 2009. 44(4): p. 1101-1106.

[17] Wong, L., W. Shen, and Y.B. Truong, Investigation of Electrospun and Film-Cast PVC Membranes Incorporated with Aliquat 336 for Efficient Cd Extraction: A

[18] Abdul-Halim, N.S., P.G. Whitten, and L.D. Nghiem, Characterising poly (vinyl

[19] Fong, H., I. Chun, and D.H. Reneker, Beaded nanofibers formed during electrospinning. Polymer, 1999. 40(16): p. 4585-4592.

[20] Huang, Z.-M., Y.Z. Zhang, M. Kotaki, and S. Ramakrishna, A review on polymer nanofibers by electrospinning and their applications in nanocomposites. Composites Science and Technology, 2003. 63(15): p. 2223-2253.

[21] Abdul Halim, N.S., P.G. Whitten, and L.D. Nghiem, The effect of aging on thermomechanical and metal extraction properties of poly (vinyl chloride)/Aliquat 336 polymer inclusion membranes. Desalination and Water Treatment, 2014: p. 1-6. 
[22] Reding, F.P., E.R. Walter, and F.J. Welch, Glass transition and melting point of poly(vinyl chloride). Journal of Polymer Science, 1962. 56(163): p. 225-231.

[23] Ribelles, J.L.G., R. Diaz-Calleja, R. Ferguson, and J.M.G. Cowie, Glass transition and physical ageing in plasticized poly(vinyl chloride). Polymer, 1987. 28(13): p. 22622266.

[24] Elicegui, A., J.J. del Val, V. Bellenger, and J. Verdu, A study of plasticization effects in poly(vinyl chloride). Polymer, 1997. 38(7): p. 1647-1657.

[25] Mikkola, J.-P., P. Virtanen, and R. Sjoholm, Aliquat 336[registered sign]-a versatile and affordable cation source for an entirely new family of hydrophobic ionic liquids. Green Chemistry, 2006. 8(3): p. 250-255.

[26] Whitten, P.G., G.M. Spinks, and G.G. Wallace, Mechanical properties of carbon nanotube paper in ionic liquid and aqueous electrolytes. Carbon, 2005. 43(9): p. 1891-1896. 


\section{$382 \quad$ Figure captions}

383 Figure 1: Schematic diagram of the experimental setup for electrospinning.

384 Figure 2: (a) Images of PVC/Aliquat 336 electron fibres (right) and PVC/Aliquat 336 PIMs

385 (left) and (b-f) surface morphology of PVC fibres at different Aliquat 336 concentration.

386 Figure 3: Surface morphology of PVC/Aliquat 336 polymer inclusion membranes at different 387 Aliquat 336 concentration.

388 Figure 4: Storage modulus curves of (a) PVC/Aliquat 336 electrospun and (b) PVC/Aliquat 389336 PIMs at different Aliquat 336 composition.

390 Figure 5: Tan $\delta$ versus temperature of (a) PVC/Aliquat 336 electrospun and (b) PVC/Aliquat 391336 PIMs.

392 Figure 6: Extraction of Cd(II) using (a) PVC/Aliquat 336 electrospuns and (B) PVC/Aliquat 393336 PIMs.

394 Figure 7: Extraction capacity against Aliquat 336 content for PVC/Aliquat 336 electrospun 395 fibres and PIMs. 
396

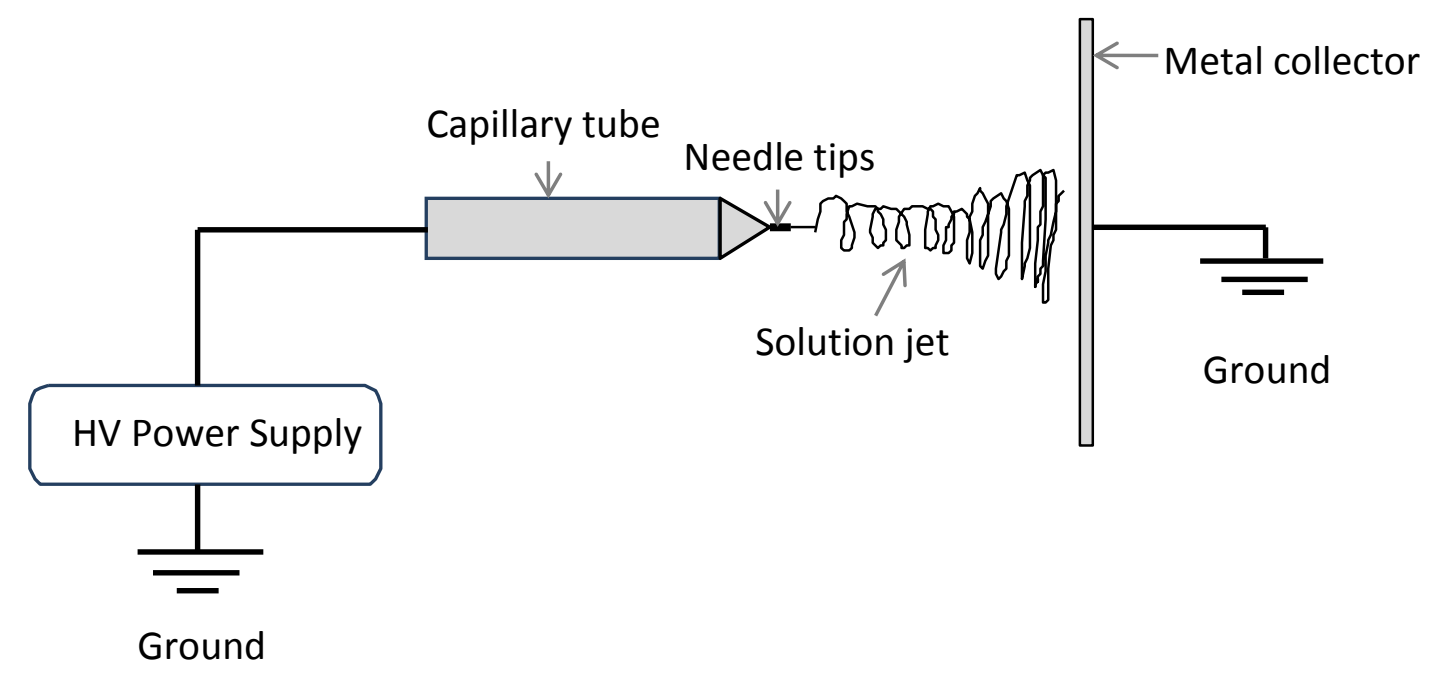

403

$404 \quad$ Figure 1 


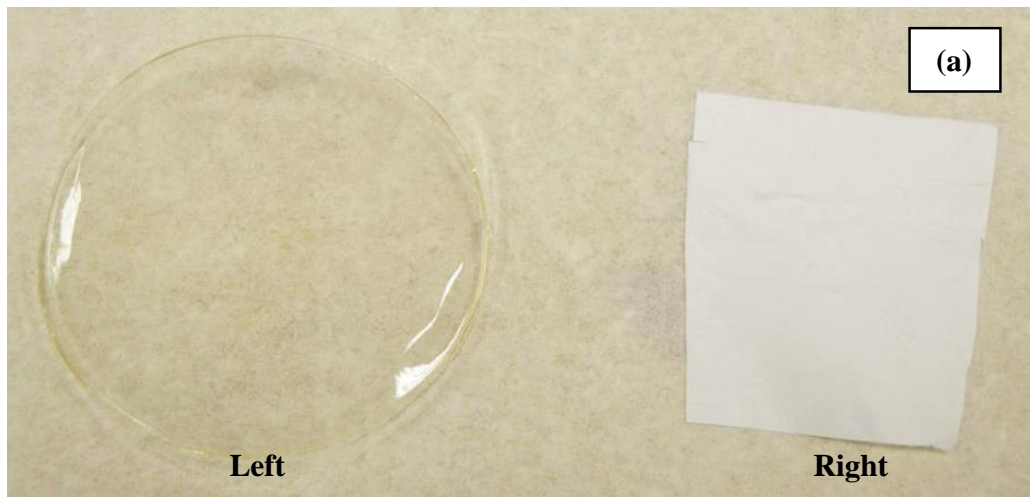

406
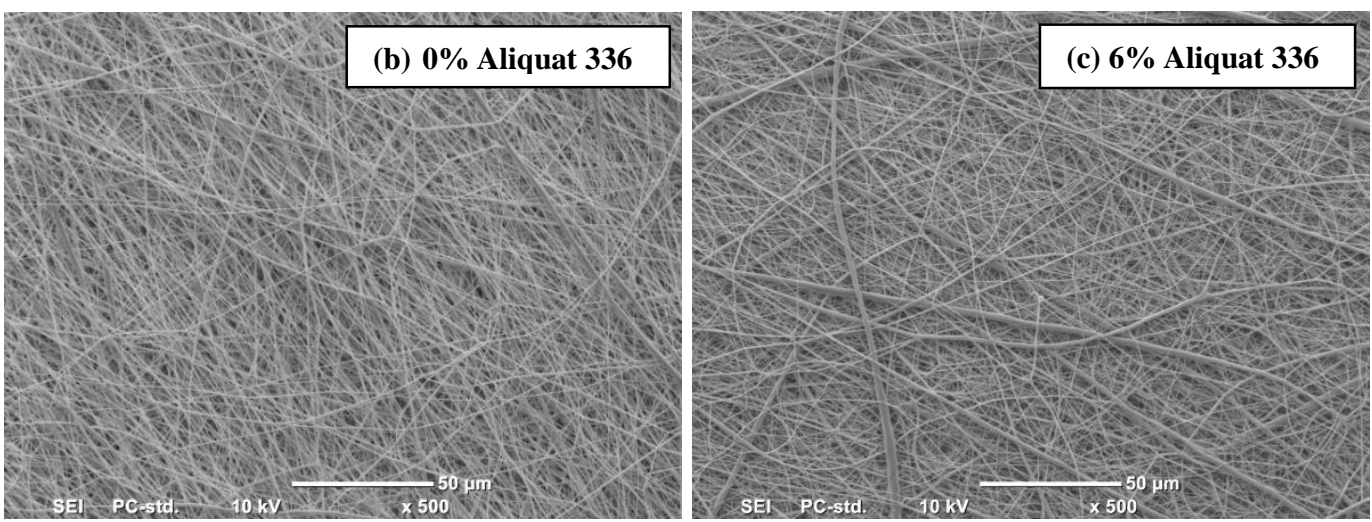

407
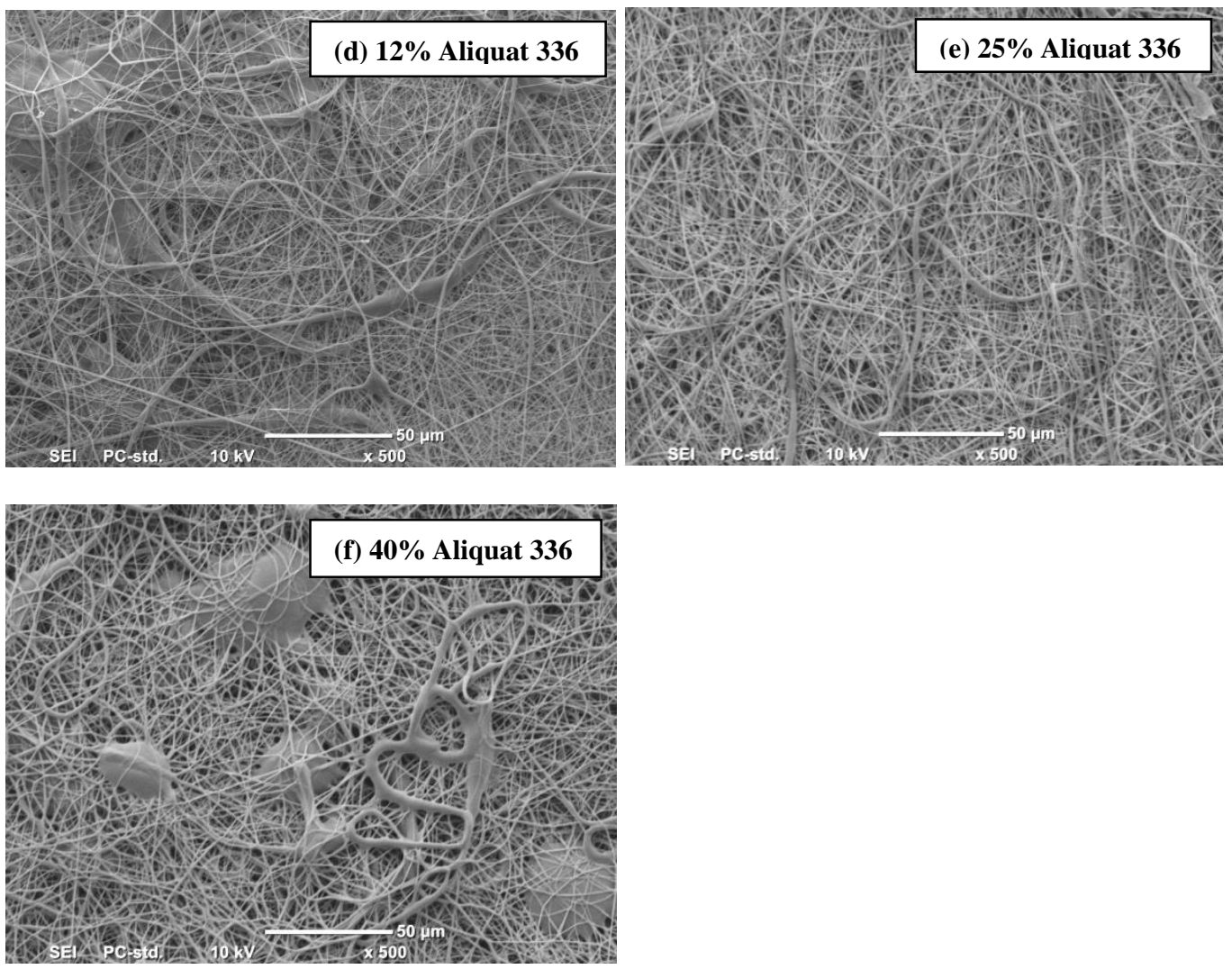

408

Figure 2 

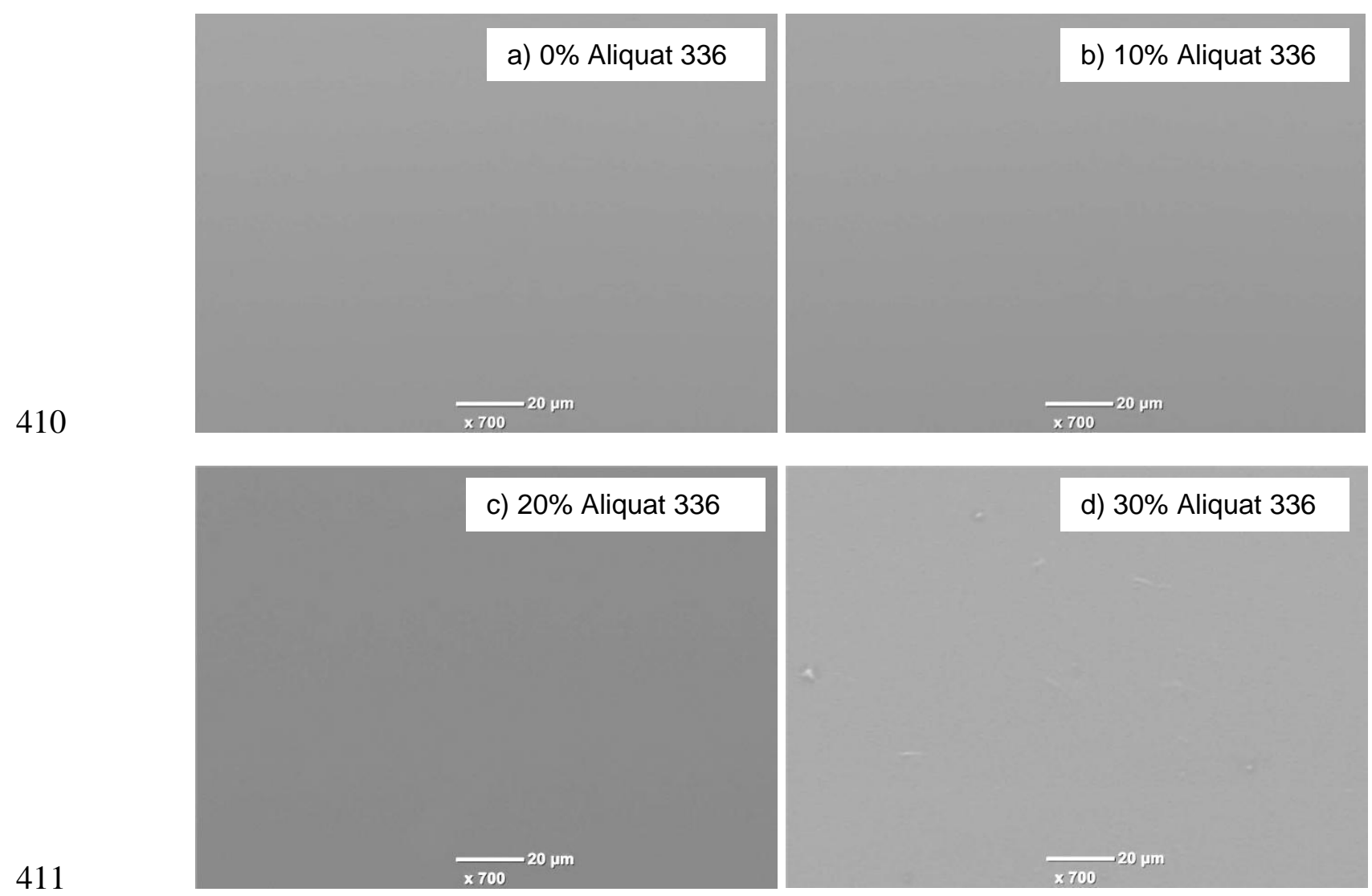

e) $40 \%$ Aliquat 336

412

$20 \mu \mathrm{m}$

413

Figure 3 


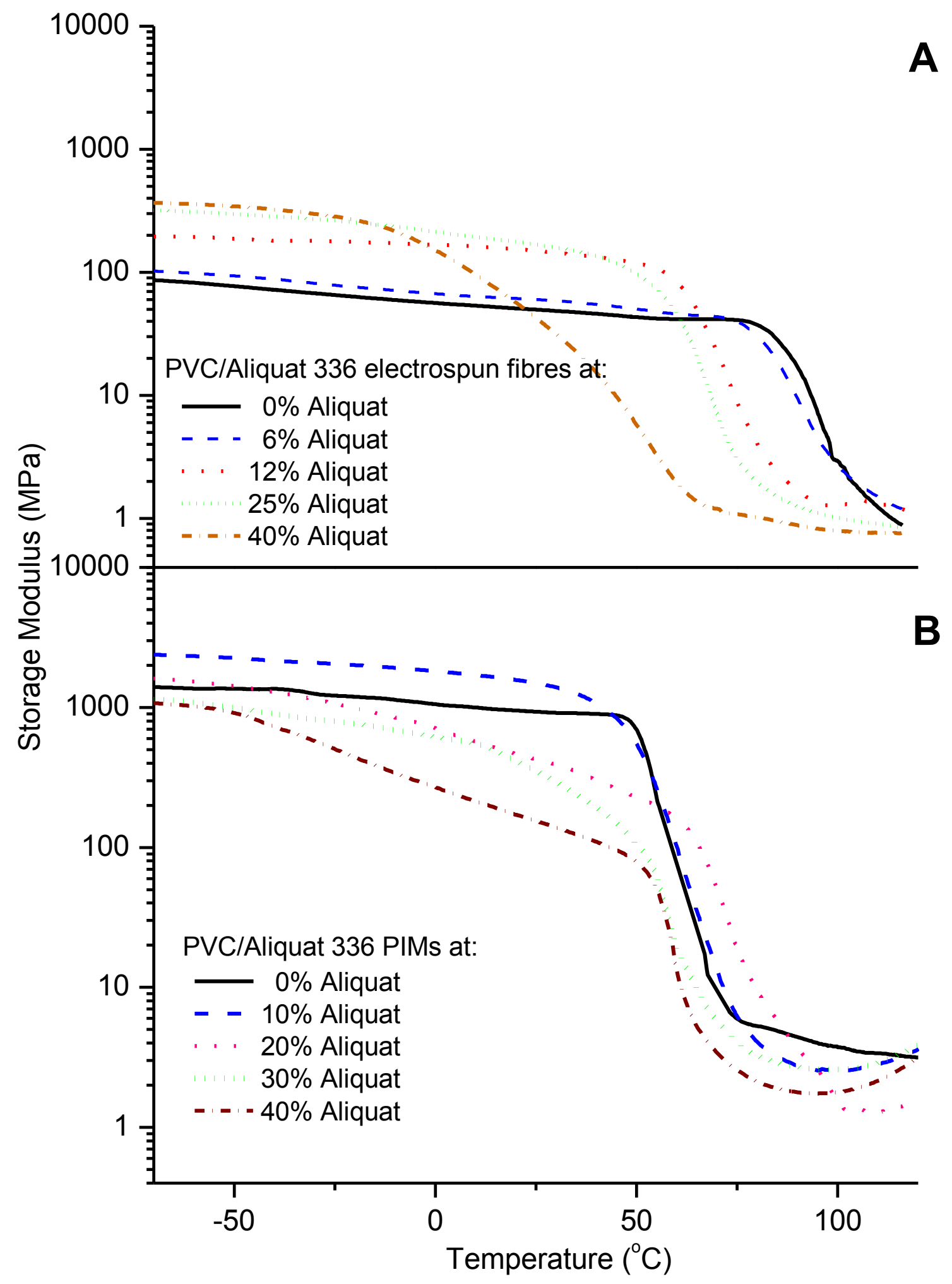

$415 \quad$ Figure 4 
A

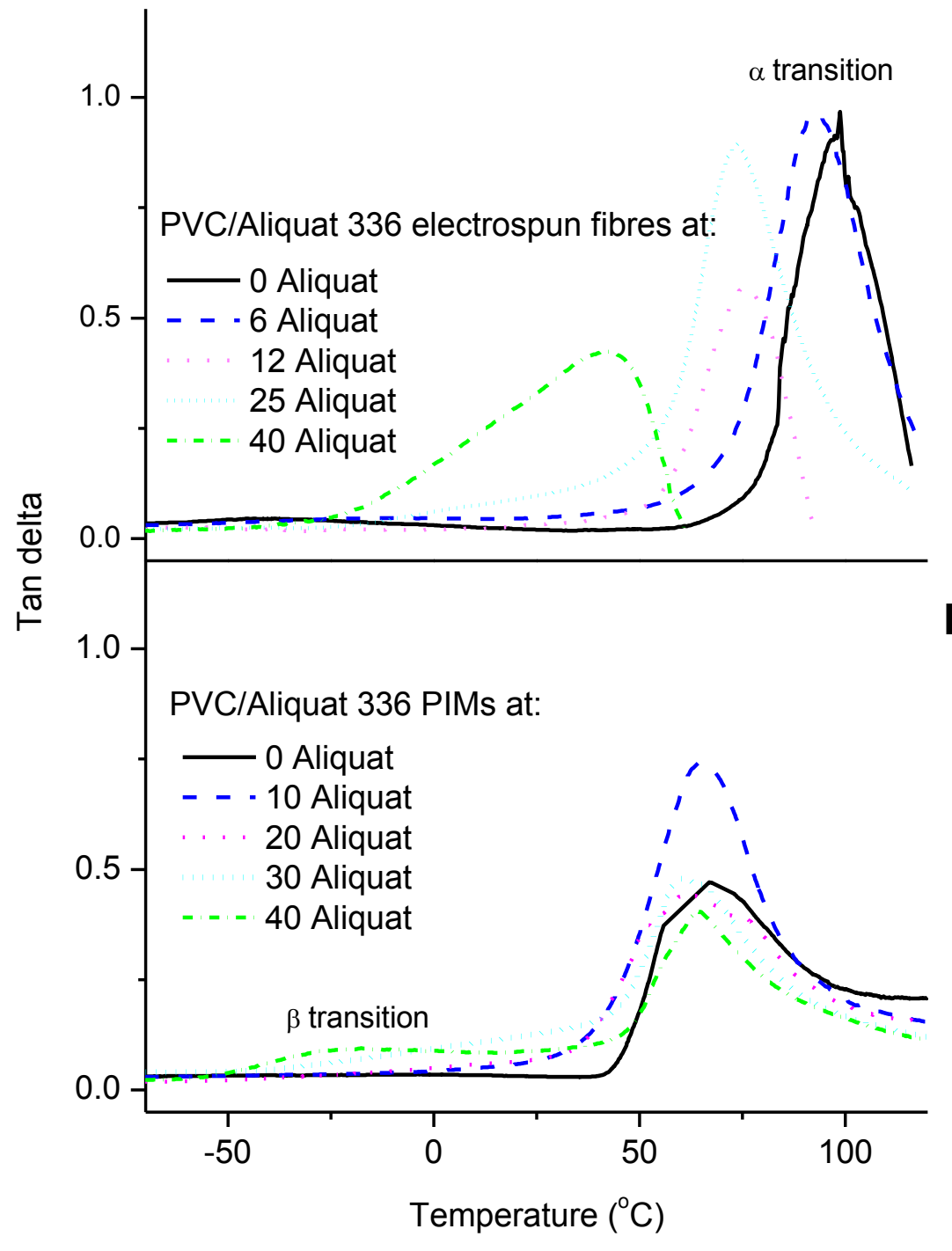

416

417 Figure 5 


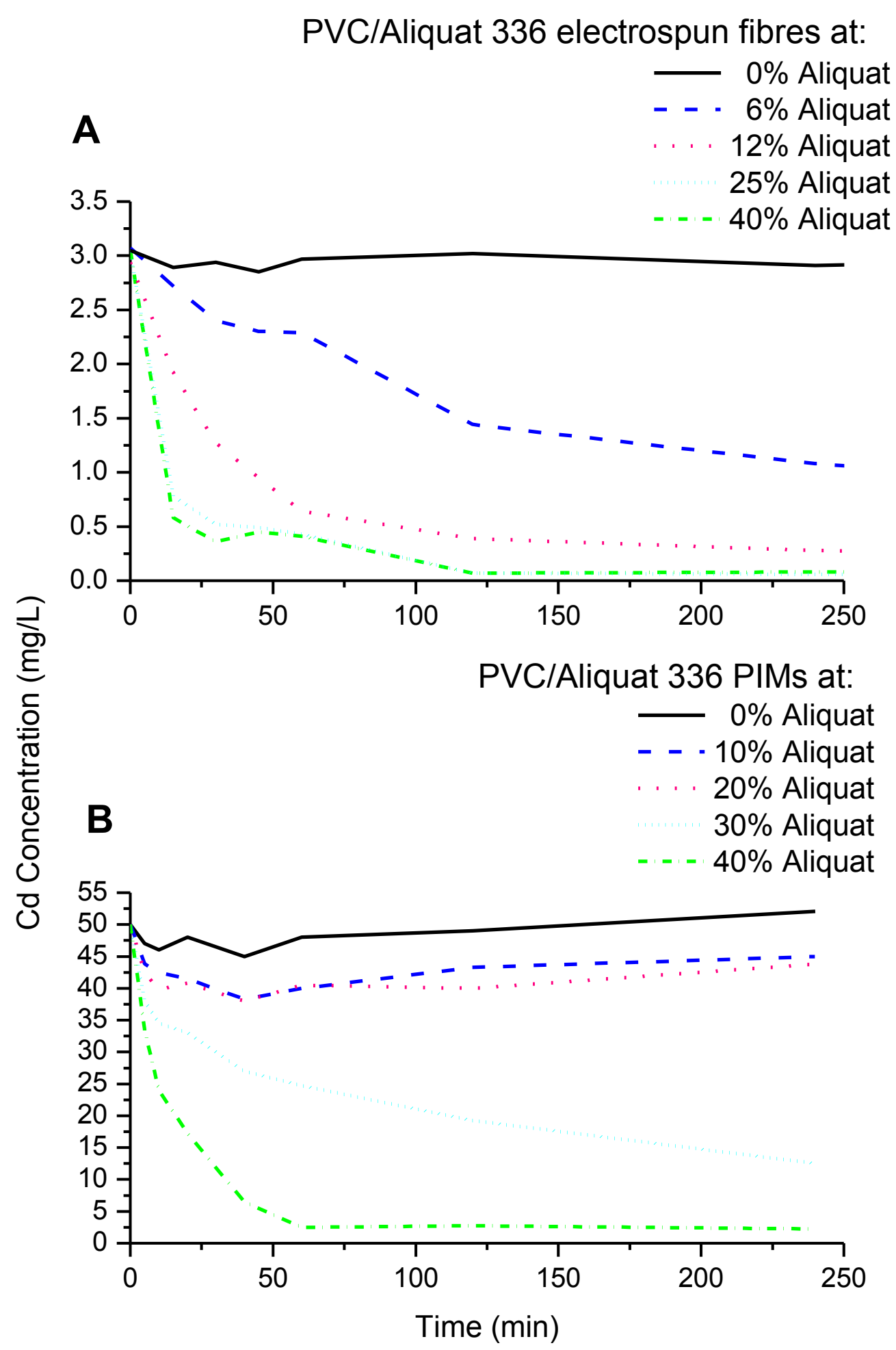

418

$419 \quad$ Figure 6 


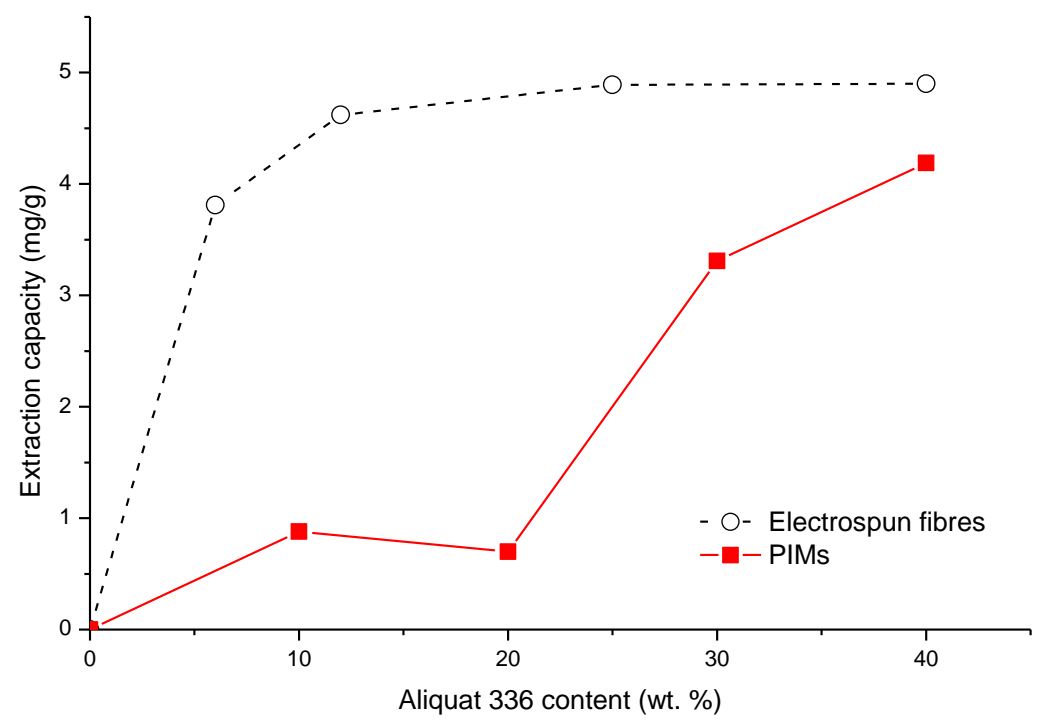

420

$421 \quad$ Figure 7 